\title{
Closing the gap in surveillance of tuberculosis and HIV co-infection, and the need for clinician-public health alliances
}

\author{
To the Editor:
}

We read with much interest the editorial by VAN DER WERF et al. [1] in the European Respiratory Journal $(E R J)$. The authors rightly advocate for the need to improve completeness, accuracy and timeliness of tuberculosis (TB)/HIV co-infection data in Europe, pledging for more cooperation between primary care physicians, medical specialists and social workers. We agree with them and others [2] that much can be gained from closer collaboration between TB and HIV control programmes, and would like to share our experience from the Netherlands. In addition, we would like to comment on the incompleteness of surveillance registers in our country, as raised by the authors.

2 years ago, we established a TB/HIV platform in the Netherlands with 16 interested clinicians and public health professionals working in TB and HIV control. The objectives for the platform were: 1) to share knowledge on both diseases; 2) to discuss management of TB/HIV patients; 3 ) to improve collaboration between professionals working in TB and HIV clinics and programmes; and 4) to systematically collect data for operational research. Table 1 provides an overview of key indicators on $\mathrm{TB} / \mathrm{HIV}$ in the Netherlands.

We identified and studied three topics.

1) HIV-co-infected TB patients: over the last 5 years, $2.9 \%$ of notified TB patients were known to have an HIV co-infection in the Netherlands [3]. We studied the records of $123 \mathrm{~TB} / \mathrm{HIV}$ patients notified in 20112015 [5]. 66 (54\%) patients had already been diagnosed with HIV prior to the time of TB diagnosis. Among this group, 29 (44\%) had been diagnosed with HIV >5 years prior to TB diagnosis, 46 (70\%) were on antiretroviral therapy and $14(21 \%)$ had a history of previous TB treatment.

2) HIV screening of TB patients: VAN DER WERF et al. [1] stated that in 2015, the proportion of TB patients with known HIV status in the Netherlands was only $60.7 \%$. They may not have been aware of our recent study of causes of unknown HIV status, published in the ERJ [6]. Our efforts to complete data entry in the National TB registry raised the proportion of TB patients with known HIV status to $73.2 \%$, all newly entered cases being HIV negative. The proportion of patients with registered HIV status in different public health authority areas ranged from $22.2 \%$ to $98.3 \%$. Our study also indicated that professionals often do not offer an HIV test to patients with a perceived nil pre-test probability and we concur with them that some patients could be exempted from testing (e.g. children born in the Netherlands because $99.8 \%$ of pregnant women are screened for HIV in the Netherlands) [7].

3) Latent tuberculosis infection (LTBI) screening of HIV-positive individuals has been a controversy in our country and, as discussed during the Wolfheze Workshops 2017, also in several other low TB incidence countries. The Dutch TB/HIV guideline has been amended after research showed that clinicians did not agree with the recommendation to screen all newly diagnosed HIV-positive individuals for LTBI [8, 9]. The current recommendation is only to screen HIV-positive individuals from high TB incidence countries or with known exposure to TB. Research is ongoing to evaluate the implementation of the guideline and the yield of LTBI screening.

Lastly, we like to comment on the incompleteness of surveillance registers. With the current electronic register, mandatory $\mathrm{TB}$ notification by clinicians and laboratories, and the national DNA fingerprinting

@ERSpublications

Collaboration between professionals working in TB and HIV clinics and programmes improves TB/ HIV surveillance http://ow.ly/sety30hXdqk

Cite this article as: de Vries G, van den Hof S, Op de Coul E, et al. Closing the gap in surveillance of tuberculosis and HIV co-infection, and the need for clinician-public health alliances. Eur Respir J 2018; 51: 1702671 [https://doi.org/10.1183/13993003.02671-2017]. 
TABLE 1 Key indicators and data on tuberculosis (TB)/HIV in the Netherlands in 2016

\begin{tabular}{lcc} 
Indicator & Number & Proportion \\
\hline TB patients notified & 889 & 5.2 per 100000 \\
Newly diagnosed individuals with HIV, projected & 816 & 4.8 per 100000 \\
Estimated individuals with HIV & 22900 & \\
Cumulative individuals with HIV diagnosed and linked to care & 20264 & $94 \%$ \\
Cumulative individuals with HIV retained in care & 19136 & $84 \%$ \\
Individuals with HIV on antiretroviral therapy & 18599 & $81 \%$ \\
TB patients with known HIV status & 620 & $70 \%$ \\
TB patients co-infected with HIV & 21 & $2.4 \%$ \\
HIV/TB patients on antiretroviral therapy & $15^{\#}$ & $71 \%$ \\
Eligible individuals with HIV screened for latent TB infection & Under study & Under study \\
Eligible individuals with HIV diagnosed with latent TB infection & Under study & Under study \\
\hline
\end{tabular}

\# : in three patients, this information was unknown $[3,4]$.

programme capturing all or nearly all Mycobacterium tuberculosis isolates, we argue that very few culture-confirmed TB cases are missed in the National TB Register in the Netherlands. Additionally, the Dutch HIV Monitoring Register, organised on a voluntarily basis, has very good national coverage [4]. The capture-recapture study [10] cited by VAN DER WERF et al. [1] may have severely overestimated nonreporting due to imperfect record linkage, which is a well-known caveat of this methodology. We are currently discussing how to close the gap between the two surveillance systems in our country and to increase our collaborative efforts to further reduce both diseases.

Gerard de Vries ${ }^{1,2}$, Susan van den Hof ${ }^{1,3,4}$, Eline Op de $\mathrm{Coul}^{2}$ and Reinout van $\mathrm{Crevel}^{5}$

${ }^{1}$ Centre for Infectious Disease Control, National Institute for Public Health and the Environment (RIVM), Bilthoven, The Netherlands. ${ }^{2}$ KNCV Tuberculosis Foundation, The Hague, The Netherlands. ${ }^{3}$ Dept of Global Health, Academic Medical Center, Amsterdam, The Netherlands. ${ }^{4}$ Amsterdam Institute for Global Health and Development, Amsterdam, The Netherlands. ${ }^{5}$ Dept of Medicine, Radboud University Medical Center, Nijmegen, The Netherlands.

Correspondence: Gerard de Vries, KNCV Tuberculosis Foundation, PO Box 146, 2501 CC Den Haag, The Netherlands. E-mail: gerard.devries@kncvtbc.org

Received: Dec 212017 | Accepted after revision: Jan 012018

Conflict of interest: None declared.

\section{References}

1 van der Werf MJ, Sotgiu G, Dara M. Closing the gap in surveillance of tuberculosis and HIV co-infection: a European perspective on the need for clinician-public health alliances. Eur Respir J 2017; 50: 1701758.

2 Lönnroth K, Migliori GB, Abubakar I, et al. Towards tuberculosis elimination: an action framework for low-incidence countries. Eur Respir J 2015; 45: 928-952.

3 Rijksinstituut voor Volksgezondheid en Milieu. Tuberculose in Nederland 2016 : Surveillancerapport inclusief rapportage monitoring van interventies [Tuberculosis in the Netherlands 2016. Surveillance and monitoring of interventions report]. www.rivm.nl/Documenten_en_publicaties/Wetenschappelijk/Rapporten/2017/december/ Tuberculose_in_Nederland_2016_Surveillancerapport_inclusief_rapportage_monitoring_van_interventies Date last updated: December 12, 2017.

4 van Sighem A, Boender S, Wit F, et al. Monitoring Report 2017. Human Immunodeficiency Virus (HIV) Infection in the Netherlands. https://www.hiv-monitoring.nl/files/1115/1117/7706/HIV_Monitoring_Report_2017. pdf Date last updated: 2017.

5 de Vries G, van Crevel R, Erkens C, et al. Tuberculose en HIV in Nederland [Tuberculosis and HIV in the Netherlands]. Tegen de Tuberculose 2017; 113: 3-6.

6 Arnoldussen M, Schimmel H, Op de Coul E, et al. Tuberculosis patients with unknown HIV status in the Netherlands: analysing underreporting and lack of testing. Eur Respir J 2017; 50: 1701257.

7 Op de Coul EL, Hahné S, van Weert YW, et al. Antenatal screening for HIV, hepatitis B and syphilis in the Netherlands is effective. BMC Infect Dis 2011; 11: 185.

8 KNCV Tuberculosis Foundation. Richtlijn Tuberculose-HIV [Tuberculosis-HIV Guideline]. https://www.kncvtbc. org/kb/3-14-richtlijn-tuberculose-hiv/ Date last updated: 2016.

9 Evenblij K, Verbon A, van Leth F. Intention of physicians to implement guidelines for screening and treatment of latent tuberculosis infection in HIV-infected patients in The Netherlands: a mixed-method design. BMC Public Health 2016; 16: 915.

10 van Leth F, Evenblij K, Wit F, et al. TB-HIV co-infection in the Netherlands: estimating prevalence and under-reporting in national registration databases using a capture-recapture analysis. J Epidemiol Community Health 2016; 70: 556-560. 